\title{
Do antidepressants interact with tamoxifen?
}

C. Pinto Ferreira ${ }^{1 *}$, J. Pereira ${ }^{2}$, I. Pinto ${ }^{1}$, M.J. Avelino ${ }^{1}$

1 - Psychiatric Hospital Centre of Lisbon, Portugal

2 - Oncology Department, West Lisbon Hospital Centre, Portugal

* Corresponding author: catarinaferreira@chpl.min-saude.pt

\section{Background:}

Breast cancer $(\mathrm{BC})$ is the most common cancer in women worldwide with 2 million new cases in 2018. Tamoxifen is a selective oestrogen receptor modulator used in patients with early stage oestrogen receptor positive $\mathrm{BC}$, reducing the risk of $\mathrm{BC}$ recurrence and death. It is an inactive pro-drug which is metabolised by the cytochrome P450(CYP)2D6 to its main active metabolite endoxifen, with 100-fold more affinity for the oestrogen receptor (Figure 1).
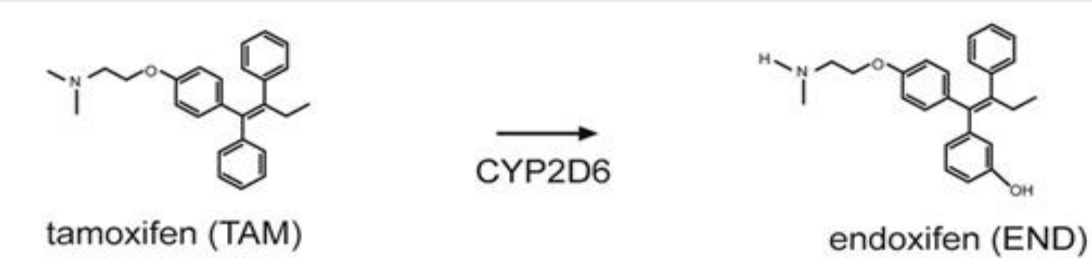

Figure 1. Tamoxifen conversion to endoxifen by CYP2D6

Aims:

The aim of this study is to systematically review the current scientific literature regarding the interactions between antidepressants and tamoxifen.
$B C$ has a known significant negative impact in women's physical and psychological wellbeing. Anxiety and depressive symptoms frequently appear, with up to one-quarter of women with $\mathrm{BC}$ being diagnosed with depressive disorder.

Newer antidepressants are an important therapeutic option to treat these conditions, being also prescribed for tamoxifen-related hot flashes. However pharmacokinetic drug interactions between hormone therapy and antidepressants may exist.

\section{Methods:}

The authors used the search engine PubMed® and selected all the articles published in English in the last ten years with the words "tamoxifen" and "antidepressants" in the title and/or abstract.

\section{Results:}

- Drugs known to be potent CYP2D6 inhibitors decrease endoxifen plasma level and compromise hormone therapy efficiency.

- Selective serotonin reuptake inhibitors (SSRIs) and serotonin and norepinephrine reuptake inhibitor (SNRIs) are commonly prescribed for treatment of depressive and anxiety disorders and they are known to inhibit CYP2D6 to varying degrees reducing endoxifen plasma levels.
- Strong CYP2D6 inhibitors include paroxetine, fluoxetine and bupropion and they must be avoided

- Paroxetine is associated to tamoxifen treatment failure, leading to a higher risk of breast cancer recurrence and increased mortality.

- The risk is related to the duration of co-exposure.
- Antidepressants that are weak CYP2D6 inhibitors do not lower the concentration of endoxifen and include venlafaxine, sertraline and citalopram

- These drugs are safer and must be rather preferred as reasonable choices.

\section{Conclusions:}

Pharmacokinetics interactions must be considered whenever prescribing antidepressant drugs in tamoxifen-treated patients since some of these psychotropic drugs can significantly impact the prognosis of cancer disease. 